\title{
Iterative scheme for the observation of a competitive Lotka-Volterra system
}

\author{
M. Gámez ${ }^{\text {a }}$ I. López ${ }^{\text {a }}$ Z. Varga ${ }^{\text {b }}$ \\ ${ }^{a}$ Department of Statistics and Applied Mathematics, University of Almería, Spain. \\ ${ }^{\mathrm{b}}$ Institute of Mathematics and Informatics, Szent István University, Gödöllö, \\ Hungary.
}

\begin{abstract}
In this work, in terms of the model parameters, sufficient conditions are established to construct a sequence of approximate observers for a two-species competitive Lotka-Volterra system. This iterative approach makes it possible to localize the solution of the system, and reveal its long-term behaviour. The main results are also illustrated by numerical simulations.
\end{abstract}

Key words: iterative scheme; local observer; competitive Lotka-Volterra system.

\section{Introduction}

As a classical description of a simple population system, the first continuoustime deterministic model of a predator-prey interaction was proposed by Lotka (1925) and Volterra (1931). This model explained certain qualitative features of the behaviour of predator-prey systems that had been observed empirically (such as the periodic change of densities with a certain delay; or the increase and decrease in the time-mean density of predator and prey fish populations, respectively, due to harvesting activity.) In Volterra (1931) a first detailed stability analysis of a generalized $n$-species model was given for the basic classes of conservative and dissipative systems. Later on, the study was extended to non Lotka-Volterra type population systems. On the other hand, in a large number of papers, in-depth analysis of particular types Lotka-Volterra systems were given. For a collection of other classical works on the subject we refer the reader to Scudo and Ziegler (1978). For further references see e. g. Freedman (1980) and Yodzis (1989).

Email addresses: mgamez@ual.es corresponding author (M. Gámez), milopez@ual.es (I. López), Varga.Zoltan@gek.szie.hu (Z. Varga). 
In this work, the Lotka-Volterra model of two competing species will be considered. For some classical results on such systems see e.g. Freedman (1980). As a more recent development, in Tineo (1996) an iterative scheme has been proposed for the study of competitive systems, see also Gámez (1999), Tineo (2001) and Carreño et al. (2002). Now we shall apply a similar iterative scheme for the construction and analysis of approximate observers for the monitoring of the state process in a two-species competitive system.

Considering a two-species competitive Lotka-Volterra model with a partially monotonous observation, first we find sufficient conditions which make it possible to construct an observer for each member of the sequence of simple auxiliary observation systems, applying the result recalled from Sundarapandian (2002). For earlier applications of observers to systems of population biology see López et al. (2007a,b,c).

Finally, we shall present results and numerical calculations for a concrete twospecies competitive system. The simulation results provide a "stripe" between two functions that is attractive for the original system in the sense that any positive solution of the latter tends to this stripe. This fact also makes it possible to make predictions on the long-term behaviour of the population system, such as coexistence (persistence) or extinction of one of the species.

\section{Preliminaries}

In this section we introduce some notation and recall some known concepts and results to be used in the paper.

\subsection{Sub-and supersolutions}

For the construction of the iterative scheme we shall use the following concepts and results. Let us consider system

$$
x^{\prime}=f(t, x),
$$

where $f: W \rightarrow \mathbb{R}^{n}$, is a locally Lipchitzian and continuous function in $x$, with an open subset $W$ of $\mathbb{R} \times \mathbb{R}^{n}$.

Definition 2.1 A continuously differentiable function $u:[a, b] \rightarrow \mathbb{R}^{n}$ is called supersolution (respectively subsolution) of system (2.1) if $(t, u(t)) \in W$ and $u^{\prime}(t) \geq f(t, u(t))$ (respectively $u^{\prime}(t) \leq f(t, u(t))$ ) for all $t \in[a, b]$. (Here $\leq$ and $\geq$ mean inequalities by coordinates.)

Now we recall a lemma of technical character for the scalar case.

Lemma 2.2 (Gámez, 1999, Corollary 1.3.8). Let $u, v:[a, b] \rightarrow \mathbb{R}$ be continuously differentiable functions such that $u$ (respectively $v$ ) is a subsolution (respectively supersolution) of(2.1) for $n=1$. If $u(a) \leq v(a)$ then $u \leq v$. 


\subsection{Construction of local observers}

Given positive integers $m, n$, we suppose that the following functions

$$
F: \mathbb{R}^{n} \rightarrow \mathbb{R}^{n}, \quad H: \mathbb{R}^{n} \rightarrow \mathbb{R}^{m}
$$

are $\mathcal{C}^{1}$ and for some $x^{*} \in \mathbb{R}^{n}$ we have that $F\left(x^{*}\right)=0$ and $H\left(x^{*}\right)=0$. We consider the following observation system

$$
\begin{aligned}
& x^{\prime}=F(x) \\
& y=H(x)
\end{aligned}
$$

where $H$ is called observation function.

Definition 2.3 A matrix $A \in \mathbb{R}^{n \times n}$ is said to be stable, if all its eigenvalues have negative real parts.

In case of $2 \times 2$ matrix the general Routh-Hurwitz criterion (see e.g. Chen (2004)) reduces to the following simple conditions, given in terms of the characteristic polynomial coefficients.

The Routh-Hurwitz criterion for $n=2$ : Let $\lambda^{2}+a_{1} \lambda+a_{2}$ be the characteristic polynomial of $A \in \mathbb{R}^{2 \times 2}$. Then $A$ is stable if and only if $a_{1}, a_{2}>0$.

Now, the construction of an observer system will be based on Sundarapandian (2002).

Definition 2.4 A $\mathcal{C}^{1}$ dynamical system described by

$$
z^{\prime}=G(z, y), \quad\left(z \in \mathbb{R}^{n}\right)
$$

is called a local asymptotic (respectively, exponential) observer for observation system (2.2)-(2.3) if the composite system (2.2)-(2.3),(2.4) satisfies the following two requirements.

i) If $x(0)=z(0)$, then $x(t)=z(t)$, for all $t \geq 0$.

ii) There exists a neighborhood $V$ of equilibrium $x^{*}$ such that for all $x(0), z(0) \in$ $V$, the estimation error $z(t)-x(t)$ decays asymptotically (respectively, exponentially) to zero.

Theorem 2.5 (Sundarapandian, 2002). Suppose that the observation system (2.2)-(2.3) is Lyapunov stable at equilibrium, and that there exists a matrix $K$ such that matrix $A-K C$ is stable, where $A:=F^{\prime}\left(x^{*}\right)$ and $C:=H^{\prime}\left(x^{*}\right)$. Then dynamic system defined by

$$
z^{\prime}=F(z)+K[y-H(z)]
$$

is a local exponential observer for observation system (2.2)-(2.3).

Remark 2.6 It is known (Sundarapandian, 2002) that under the condition

$$
\operatorname{rank}\left[C|C A| C A^{2}|\ldots| C A^{n-1}\right]^{T}=n
$$


for local observability, the existence of such an observer is guaranteed. The above theorem provides an efficient method to construct this observer.

\section{Iterative scheme}

We shall consider a so-called competitive Lotka-Volterra system

$$
x_{i}^{\prime}=x_{i}\left(a_{i}-\sum_{j=1}^{2} b_{i j} x_{j}\right)=: x_{i} f_{i}\left(x_{1}, x_{2}\right), \quad i=1,2
$$

with $a_{i}, b_{i j}>0$ for all $i, j=1,2$, satisfying condition,

$$
a_{1} b_{22}-b_{12} a_{2}>0 ; a_{2} b_{11}-b_{21} a_{1}>0 ; b_{11} b_{22}-b_{21} b_{12}>0 \text {. }
$$

Let $h: \mathbb{R}^{2} \rightarrow \mathbb{R}$ be a locally bounded function with partial derivatives $h_{x_{i}}>0$ for $i=1,2$, defined and continuous in $\mathbb{R}^{2}$. Let us consider the following observation system

$$
\begin{aligned}
x_{1}^{\prime} & =x_{1}\left(a_{1}-b_{11} x_{1}-b_{12} x_{2}\right) \\
x_{2}^{\prime} & =x_{2}\left(a_{2}-b_{21} x_{1}-b_{22} x_{2}\right) \\
y & =h\left(x_{1}, x_{2}\right)-h\left(x_{1}^{*}, x_{2}^{*}\right) .
\end{aligned}
$$

A straightforward calculation shows that under condition $(C 1)$, system (3.1) has an asymptotically stable positive equilibrium $x^{*}=\left(x_{1}^{*}, x_{2}^{*}\right)$ (see e.g. Freedman, 1980).

Our objective is to determine sufficient conditions, in order to find a sequence of observation systems, which provides a long-term upper and lower estimation of the solutions of system (3.1). To this end, let us introduce the following concepts and notation. Given a natural number $N$, let $x^{* i}$ be the positive equilibrium of system

$$
\begin{aligned}
& x_{1}^{\prime}=x_{1} f_{1}\left(x_{1}, x_{2}^{*(i-1)}\right) \\
& x_{2}^{\prime}=x_{2} f_{2}\left(x_{1}^{*(i-1)}, x_{2}\right)
\end{aligned} \quad i=1,2, \ldots, N
$$

with $x^{* 0}=(0,0)$. For $i=1,2, \ldots, N$ we define, the following observation systems $\left(O S_{i}\right)$ :

$$
\begin{aligned}
& x_{1}^{\prime}=x_{1} f_{1}\left(x_{1}, x_{2}^{*(i-1)}\right) \\
& x_{2}^{\prime}=x_{2} f_{2}\left(x_{1}^{*(i-1)}, x_{2}\right) \\
& y^{i}=h^{i}\left(x_{1}, x_{2}\right):=h\left(x_{1}, x_{2}\right)-h\left(x_{1}^{* i}, x_{2}^{* i}\right) .
\end{aligned}
$$

We note that here and throughout the paper, upper indices are not exponents. 
Proposition 3.1 Given $k_{1}, k_{2}>0$ we have

$$
0<U^{2} \leq U^{4} \leq \ldots \leq U^{2 N} \leq U^{2 N-1} \leq \ldots \leq U^{3} \leq U^{1},
$$

where $U^{N}(N \in \mathbb{N})$ is the solution of the initial value problem

$$
\begin{aligned}
z_{1}^{\prime}= & z_{1} f_{1}\left(z_{1}, x_{2}^{*(N-1)}\right)+k_{1}\left(y^{N}-h^{N}\left(z_{1}, z_{2}\right)\right) \\
z_{2}^{\prime}= & z_{2} f_{1}\left(x_{1}^{*(N-1)}, z_{2}\right)+k_{2}\left(y^{N}-h^{N}\left(z_{1}, z_{2}\right)\right) \\
& z^{0}=\left(z_{1}^{0}, z_{2}^{0}\right) .
\end{aligned}
$$

Proof. We shall follow an inductive process:

Step 1. In system (3.1), we set $x_{2}:=0$ in $f_{1}$ and $x_{1}:=0$ in $f_{2}$, and consider the observation system $\left(O S_{1}\right)$

$$
\begin{aligned}
& x_{1}^{\prime}=F_{1}^{1}(x)=x_{1} f_{1}^{1}\left(x_{1}, x_{2}\right):=x_{1} f_{1}\left(x_{1}, 0\right)=x_{1}\left(a_{1}-b_{11} x_{1}\right) \\
& x_{2}^{\prime}=F_{2}^{1}(x)=x_{2} f_{2}^{1}\left(x_{1}, x_{2}\right):=x_{2} f_{2}\left(0, x_{2}\right)=x_{2}\left(a_{2}-b_{22} x_{2}\right) \\
& y^{1}=h^{1}\left(x_{1}, x_{2}\right):=h\left(x_{1}, x_{2}\right)-h\left(x_{1}^{* 1}, x_{2}^{* 1}\right)
\end{aligned}
$$

where $x_{1}^{* 1}=\frac{a_{1}}{b_{11}} ; x_{2}^{* 1}=\frac{a_{2}}{b_{22}}$. It is easy to check that $x^{* 1}>x^{*}$. Now, with matrices

$$
\begin{aligned}
& A^{1}:=\frac{\partial F^{1}}{\partial x}\left(x^{* 1}\right)=\left(\begin{array}{cc}
a_{1}-2 b_{11} x_{1}^{* 1} & 0 \\
0 & a_{2}-2 b_{22} x_{2}^{* 1}
\end{array}\right) \\
& C^{1}=\left(c_{1}^{1}, c_{2}^{1}\right):=\left(\frac{\partial h^{1}}{\partial x_{1}}\left(x^{* 1}\right), \frac{\partial h^{1}}{\partial x_{2}}\left(x^{* 1}\right)\right)
\end{aligned}
$$

and $K=\operatorname{col}\left(k_{1}, k_{2}\right)$ we calculate the characteristical polynomial of the auxiliary matrix $A^{1}-K C^{1}$ :

$$
\begin{aligned}
p^{1}(\lambda) & =\lambda^{2}+\left(a_{1}+a_{2}+k_{1} c_{1}^{1}+k_{2} c_{2}^{1}\right) \lambda+\left(a_{1}+k_{1} c_{1}^{1}\right)\left(a_{2}+k_{2} c_{2}^{1}\right)-k_{1} k_{2} c_{1}^{1} c_{2}^{1} \\
& =\lambda^{2}+\left(a_{1}+a_{2}+k_{1} c_{1}^{1}+k_{2} c_{2}^{1}\right) \lambda+a_{1} a_{2}+a_{1} k_{2} c_{2}^{1}+a_{2} k_{1} c_{1}^{1} .
\end{aligned}
$$

As $h_{x_{i}}>0$ for $i=1,2$ and $a_{1}, a_{2}, k_{1}, k_{2}>0$ we obtain that

$$
a_{1}+a_{2}+k_{1} c_{1}^{1}+k_{2} c_{2}^{1}>0 \quad ; \quad a_{1} a_{2}+a_{1} k_{2} c_{2}^{1}+a_{2} k_{1} c_{1}^{1}>0
$$

therefore, according to the Routh-Hurwitz criterion, matrix $A^{1}-K C^{1}$ is stable, and by Theorem 2.5 we can construct the observer of system (3.6) in the following way:

$$
\begin{aligned}
& z_{1}^{\prime}=z_{1}\left(a_{1}-b_{11} z_{1}\right)+k_{1}\left(y^{1}-h^{1}(z)\right) \\
& z_{2}^{\prime}=z_{2}\left(a_{2}-b_{22} z_{2}\right)+k_{2}\left(y^{1}-h^{1}(z)\right)
\end{aligned}
$$


Given an initial condition $z^{01}=\left(z_{1}^{01}, z_{2}^{01}\right)$ near $x^{* 1}$, we can find a solution of (3.7) denoted by $U^{1}$.

Step 2. Now, in system (3.1), we set $x_{2}:=x_{2}^{* 1}$ in $f_{1}$ and $x_{1}:=x_{1}^{* 1}$ in $f_{2}$, defining observation system $\left(O S_{2}\right)$

$$
\begin{aligned}
& x_{1}^{\prime}=F_{1}^{2}(x)=x_{1} f_{1}^{2}\left(x_{1}, x_{2}\right):=x_{1} f_{1}\left(x_{1}, x_{2}^{* 1}\right)=x_{1}\left(a_{1}-b_{11} x_{1}-b_{12} x_{2}^{* 1}\right) \\
& x_{2}^{\prime}=F_{2}^{2}(x)=x_{2} f_{2}^{2}\left(x_{1}, x_{2}\right):=x_{2} f_{2}\left(x_{1}^{* 1}, x_{2}\right)=x_{2}\left(a_{2}-b_{21} x_{1}^{* 1}-b_{22} x_{2}\right) \\
& y^{2}=h^{2}\left(x_{1}, x_{2}\right):=h\left(x_{1}, x_{2}\right)-h\left(x_{1}^{* 2}, x_{2}^{* 2}\right)
\end{aligned}
$$

where $x_{1}^{* 2}=\frac{a_{1}-b_{12} x_{2}^{* 1}}{b_{11}}>0 ; x_{2}^{* 2}=\frac{a_{2}-b_{21} x_{1}^{* 1}}{b_{22}}>0$, which are positive by $(C 1)$, moreover, inequalities $x^{* 1}>x^{*}>x^{* 2}$ hold.

Again, with matrices

$$
\begin{aligned}
A^{2} & :=\frac{\partial F^{2}}{\partial x}\left(x^{* 2}\right)=\left(\begin{array}{cc}
a_{1}-2 b_{11} x_{1}^{* 2}-b_{12} x_{2}^{* 1} & 0 \\
0 & a_{2}-b_{21} x_{1}^{* 1}-2 b_{22} x_{2}^{* 2}
\end{array}\right) \\
C^{2} & :=\left(c_{1}^{2}, c_{2}^{2}\right):=\left(\frac{\partial h^{2}}{\partial x_{1}}\left(x^{* 2}\right), \frac{\partial h^{2}}{\partial x_{2}}\left(x^{* 2}\right)\right),
\end{aligned}
$$

if we calculate the characteristical polynomial of $A^{2}-K C^{2}$,

$$
\begin{aligned}
p^{2}(\lambda)= & \lambda^{2}+\left(b_{11} x_{1}^{* 2}+b_{22} x_{2}^{* 2}+k_{1} c_{1}^{2}+k_{2} c_{2}^{2}\right) \lambda+ \\
& +b_{11} b_{22} x_{1}^{* 2} x_{2}^{* 2}+b_{11} x_{1}^{* 2} k_{2} c_{2}^{2}+b_{22} x_{2}^{* 2} k_{1} c_{1}^{2}
\end{aligned}
$$

by Routh-Hurwitz criterion, we again have that $A^{2}-K C^{2}$ is stable and we can construct the observer of system (3.8)

$$
\begin{aligned}
& z_{1}^{\prime}=z_{1}\left(a_{1}-b_{11} z_{1}-b_{12} x_{2}^{* 1}\right)+k_{1}\left(y^{2}-h^{2}(z)\right) \\
& z_{2}^{\prime}=z_{2}\left(a_{2}-b_{21} x_{1}^{* 1}-b_{22} z_{2}\right)+k_{2}\left(y^{2}-h^{2}(z)\right) .
\end{aligned}
$$

Now, for an initial condition $z^{02}=\left(z_{1}^{02}, z_{2}^{02}\right)$ near $x^{* 2}$ with $z^{02} \leq z^{01}$, we find a solution $U^{2}$ of (3.9). Moreover, by the partial monotony of $h$, we have

$$
y^{1}-h^{1}(z)>y^{2}-h^{2}(z)
$$

which permit us to compare the solution of systems (3.7) and (3.9) with initial conditions $z^{02} \leq z^{01}$. Hence we get

$$
U^{2} \leq U^{1}
$$


Step 3. In system (3.1), we set $x_{2}:=x_{2}^{* 2}$ in $f_{1}$ and $x_{1}:=x_{1}^{* 2}$ in $f_{2}$. Hence we obtain observation system $\left(O S_{3}\right)$

$$
\begin{aligned}
& x_{1}^{\prime}=F_{1}^{3}(x)=x_{1}\left(a_{1}-b_{11} x_{1}-b_{12} x_{2}^{* 2}\right)=x_{1} f_{1}\left(x_{1}, x_{2}^{* 2}\right) \\
& x_{2}^{\prime}=F_{2}^{3}(x)=x_{2}\left(a_{2}-b_{21} x_{1}^{* 2}-b_{22} x_{2}\right)=x_{2} f_{2}\left(x_{1}^{* 2}, x_{2}\right) \\
& y^{3}=h^{3}\left(x_{1}, x_{2}\right):=h\left(x_{1}, x_{2}\right)-h\left(x_{1}^{* 3}, x_{2}^{* 3}\right)
\end{aligned}
$$

where $x_{1}^{* 3}=\frac{a_{1}-b_{12} x_{2}^{* 2}}{b_{11}}>0 ; x_{2}^{* 3}=\frac{a_{2}-b_{21} x_{1}^{* 2}}{b_{22}}>0$, which are positive by $(C 1)$, moreover, inequalities $x^{* 1}>x^{* 3}>x^{*}>x^{* 2}$ are verified.

If we calculate the characteristic polynomial of matrix $A^{3}-K C^{3}$ with

$$
\begin{aligned}
& A^{3}:=\frac{\partial F^{3}}{\partial x}\left(x^{* 3}\right)=\left(\begin{array}{cc}
a_{1}-2 b_{11} x_{1}^{* 3}-b_{12} x_{2}^{* 2} & 0 \\
0 & a_{2}-b_{21} x_{1}^{* 2}-2 b_{22} x_{2}^{* 3}
\end{array}\right) \\
& C^{3}:=\left(c_{1}^{3}, c_{2}^{3}\right):=\left(\frac{\partial h^{3}}{\partial x_{1}}\left(x^{* 3}\right), \frac{\partial h^{3}}{\partial x_{2}}\left(x^{* 3}\right)\right),
\end{aligned}
$$

according to the Routh-Hurwitz criterion we get that $A^{3}-K C^{3}$ is stable, therefore we can construct the observer of system (3.10) in the following way:

$$
\begin{aligned}
& z_{1}^{\prime}=z_{1}\left(a_{1}-b_{11} z_{1}-b_{12} x_{2}^{* 2}\right)+k_{1}\left(y^{3}-h^{3}(z)\right) \\
& z_{2}^{\prime}=z_{2}\left(a_{2}-b_{21} x_{1}^{* 2}-b_{22} z_{2}\right)+k_{2}\left(y^{3}-h^{3}(z)\right),
\end{aligned}
$$

and for an initial condition $z^{03}=\left(z_{1}^{03}, z_{2}^{03}\right)$ near $x^{* 3}$, such that $z^{02} \leq z^{03} \leq z^{01}$, we can find a solution denoted by $U^{3}$. Moreover, again by the strict partial monotony of $h$ we have

$$
y^{1}-h^{1}(z)>y^{3}-h^{3}(z)>y^{2}-h^{2}(z) .
$$

Now comparing the solutions of systems (3.7), (3.9) and (3.11), we obtain

$$
0<U^{2} \leq U^{3} \leq U^{1}
$$

The process can be continued in an iterative way, which completes the proof.

From the above construction we have two sequences $\left(U^{2 N-1}\right)$ and $\left(U^{2 N}\right)$ for $N \in \mathbb{N}$ that are monotonous and bounded, therefore, convergent point-wise. Hence, for each $t \in \mathbb{R}$, we define:

$$
\bar{U}(t)=\lim _{N \rightarrow+\infty} U^{2 N-1}(t) \quad, \quad \underline{U}(t)=\lim _{N \rightarrow+\infty} U^{2 N}(t) .
$$


Moreover, as

$$
\begin{aligned}
& \left(U_{1}^{N}\right)^{\prime}=U_{1}^{N}\left(a_{1}-b_{11} U_{1}^{N}-b_{12} x_{2}^{*(N-1)}\right)+k_{1}\left(y^{N}-h^{N}\left(U^{N}\right)\right) \\
& \left(U_{2}^{N}\right)^{\prime}=U_{2}^{N}\left(a_{2}-b_{21} x_{1}^{*(N-1)}-b_{22} U_{2}^{N}\right)+k_{2}\left(y^{N}-h^{N}\left(U^{N}\right)\right)
\end{aligned}
$$

and $h$ is bounded on compact sets, sequence $\left(\left(U^{N}\right)^{\prime}\right)$ is uniformly bounded on compact sets. Applying the Ascoli Theorem to a fixed compact subset of $[0,+\infty)$, this sequence has a uniformly convergent subsequence denoted in the same way. By (3.4) we conclude that the convergence expressed in (3.12) is uniform on compact sets of $\mathbb{R}$.

On the other hand, the idea developed in the above iterative process, also applies to a positive solution of (3.1). In this way we can also establish a relation between the two iterative schemes and as a result, we shall conclude that every solution of system (3.1) is attracted by the "stripe" $[\underline{U}, \bar{U}]$.

Indeed, let $u=\left(u_{1}, u_{2}\right)$ be the positive solution of system (3.1), satisfying the initial condition $x^{0}>0$, near $x^{*}$. Define $x_{2}^{* 0}:=0$ and substitute it as $x_{2}$ in $f_{1}\left(x_{1}, x_{2}\right)$ of system $(3.1)$, to obtain

$$
x_{1}^{\prime}=x_{1}\left(a_{1}-b_{11} x_{1}\right) \quad x_{1}(0)=x_{1}^{0} .
$$

This initial value problem has a solution $u_{1}^{1}$ defined and bounded in $[0,+\infty)$. Moreover, by the monotony of $f_{1}$, we have $f_{1}\left(x_{1}, u_{2}\right) \leq f_{1}\left(x_{1}, 0\right)$ implying $u_{1}^{1}$ is a supersolution of $x_{1}^{\prime}=x_{1} f_{1}\left(x_{1}, u_{2}\right)$, and by Lemma 2.2 we have $u_{1} \leq u_{1}^{1}$.

We define $x_{1}^{* 0}:=0$ and substitute it as $x_{1}$ in $f_{2}\left(x_{1}, x_{2}\right)$ of system (3.1) to obtain

$$
x_{2}^{\prime}=x_{2}\left(a_{2}-b_{22} x_{2}\right) \quad x_{2}(0)=x_{2}^{0}
$$

which has a solution $u_{2}^{1}$ defined and bounded in $[0,+\infty)$. Moreover, by the monotony of $f_{2}$, we have $f_{2}\left(u_{1}, x_{2}\right) \leq f_{2}\left(0, x_{2}\right)$, therefore $u_{2}^{1}$ is a supersolution of $x_{2}^{\prime}=x_{2} f_{2}\left(u_{1}, x_{2}\right)$, and by Lemma 2.2 , we obtain $u_{2} \leq u_{2}^{1}$.

Now we take $x_{2}^{* 1}$ defined in (3.3) and substitute it into $f_{1}\left(x_{1}, x_{2}\right)$, obtaining the initial value problem:

$$
x_{1}^{\prime}=x_{1}\left(a_{1}-b_{11} x_{1}-b_{12} x_{2}^{* 1}\right) \quad x_{1}(0)=x_{1}^{0},
$$

which has a solution $u_{1}^{2}$ defined and bounded in $[0,+\infty)$. Moreover, by the monotony of $f_{1}$, we have $f_{1}\left(x_{1}, u_{2}\right) \geq f_{1}\left(x_{1}, x_{2}^{* 1}\right)$, therefore $u_{1}^{2}$ is a subsolution of $x_{1}^{\prime}=x_{1} f_{1}\left(x_{1}, u_{2}\right)$, and by Lemma 2.2 , we have $u_{1} \geq u_{1}^{2}$. If we take $x_{1}^{* 1}$ defined in (3.3), and substitute it into $f_{2}\left(x_{1}, x_{2}\right)$, we get the initial value problem

$$
x_{2}^{\prime}=x_{2}\left(a_{2}-b_{21} x_{1}^{* 1}-b_{22} x_{2}\right) \quad x_{2}(0)=x_{2}^{0},
$$

which has a solution $u_{2}^{2}$. Now, similarly to the reasoning made for $u_{1}^{2}$, we obtain $u_{2} \geq u_{2}^{2}$. Therefore,

$$
u^{2}(t) \leq u(t) \leq u^{1}(t), \quad \forall t \geq 0
$$


If we continue the process, we get a sequence of solutions, that in general terms we can express by the following iterative scheme:

$$
\begin{array}{ll}
\left(u_{1}^{N}\right)^{\prime}=u_{1}^{N}\left(a_{1}-b_{11} u_{1}^{N}-b_{12} u_{2}^{*(N-1)}\right) & u_{1}^{N}(0)=x_{1}^{0} \\
\left(u_{2}^{N}\right)^{\prime}=u_{2}^{N}\left(a_{2}-b_{21} u_{1}^{*(N-1)}-b_{22} u_{2}^{N}\right) & u_{2}^{N}(0)=x_{2}^{0}
\end{array}
$$

with $N \in \mathbb{N}$ and $u_{1}^{* 0}=u_{2}^{* 0}=0$. Moreover, it is easy to check that the following monotony conditions are satisfied,

$$
0 \leq u^{2} \leq u^{4} \leq \ldots \leq u^{2 N} \leq u \leq u^{2 N-1} \leq \ldots \leq u^{3} \leq u^{1} .
$$

From the definition of observer, we immediately obtain

Proposition 3.2 Given $N \in \mathbb{N}$, for $u^{N}, U^{N}$ defined in the iterative schemes (3.14) and (3.5), we have

$$
u^{N}(t)-U^{N}(t) \rightarrow 0 \text { when } t \rightarrow+\infty .
$$

Remark 3.3 Although from the iterative scheme Ascoli's Theorem provides only a sequence $\left(U^{N}\right)$ uniformly convergent on compact sets, our simulation results suggest that this convergence is uniform on $[0,+\infty)$, too. As a matter of fact, as we illustrate in the next section, we also observe that if $u$ is a positive solution of system (3.1), then for all $\epsilon>0$ there exists $t_{0} \geq 0$ such that

$$
\underline{U}_{i}(t)-\epsilon \leq u_{i}(t) \leq \bar{U}_{i}(t)+\epsilon \quad i=1,2 ; \quad t \geq t_{0} .
$$

\section{Example}

For system (3.1) we consider the observed function $h: \mathbb{R}^{2} \rightarrow \mathbb{R}$ defined by $h\left(x_{1}, x_{2}\right)=x_{1}+x_{2}$, which is increasing in $x_{1}$ and $x_{2}$. Therefore, if we take $k_{1}, k_{2}>0$, the hypothesis of Proposition 3.1 is verified and the inequalities of (3.4) hold. We take the observation system

$$
\begin{aligned}
x_{1}^{\prime} & =x_{1}\left(1-0.9 x_{1}-0.5 x_{2}\right) \\
x_{2}^{\prime} & =x_{2}\left(1-0.5 x_{1}-0.7 x_{2}\right) \\
y & =h\left(x_{1}, x_{2}\right)-h\left(x_{1}^{*}, x_{2}^{*}\right),
\end{aligned}
$$

where $x^{*}=\left(x_{1}^{*}, x_{2}^{*}\right)$ is the equilibrium of system

$$
\begin{aligned}
& x_{1}^{\prime}=x_{1}\left(1-0.9 x_{1}-0.5 x_{2}\right) \\
& x_{2}^{\prime}=x_{2}\left(1-0.5 x_{1}-0.7 x_{2}\right) .
\end{aligned}
$$

For $k_{1}=k_{2}=0.5>0$ and $z^{0}=(1.4,3.9)$, we solve numerically observation systems $O S_{i}$ for $i=1, \ldots, 4$, observing how inequalities of (3.4) hold (see 
Figures 1-2), that is

$$
U^{1} \geq U^{3} \geq U^{4} \geq U^{2}
$$

Moreover, as we have mentioned in the previous section, we can check that from certain $t \in \mathbb{R}^{+}$we have that the solution $u$ of system (4.2) verifies

$$
U^{1} \geq U^{3} \geq u \geq U^{4} \geq U^{2} .
$$

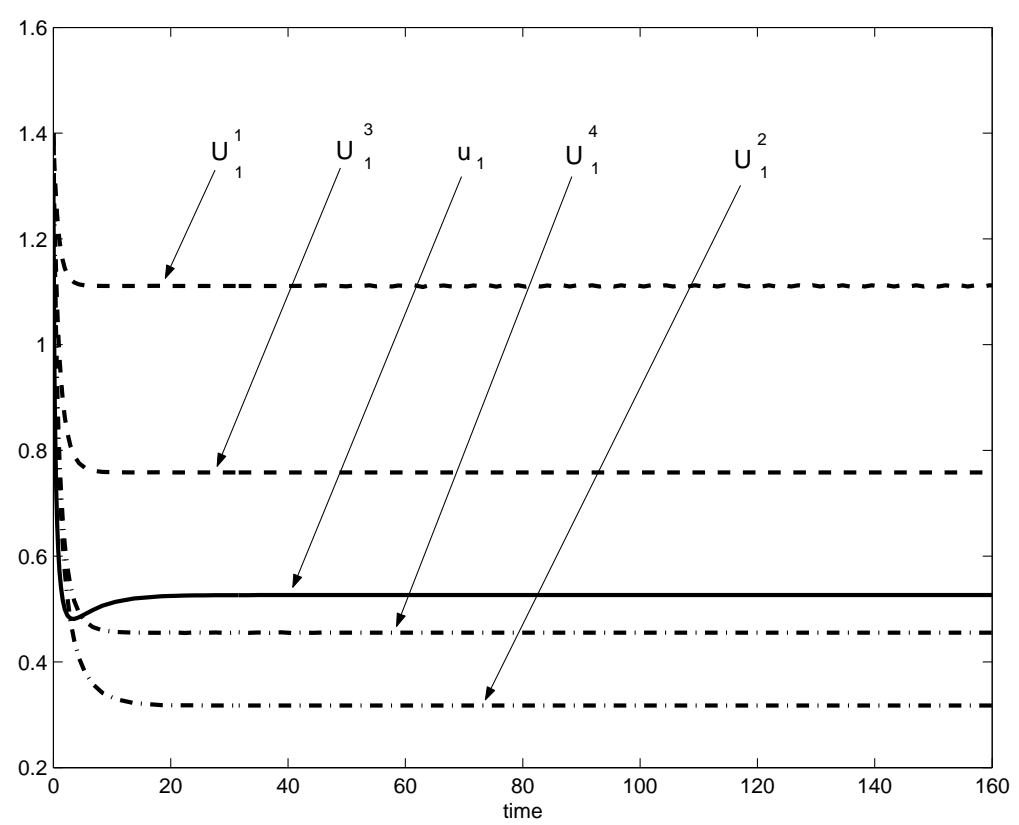

Fig. 1. Iterative scheme for $z^{0}=(1.4,3.9)$

If we solve the above observation system numerically for any other positive initial condition and arbitrary coefficients from the parameter space of the model, we obtain the same convergence result. 


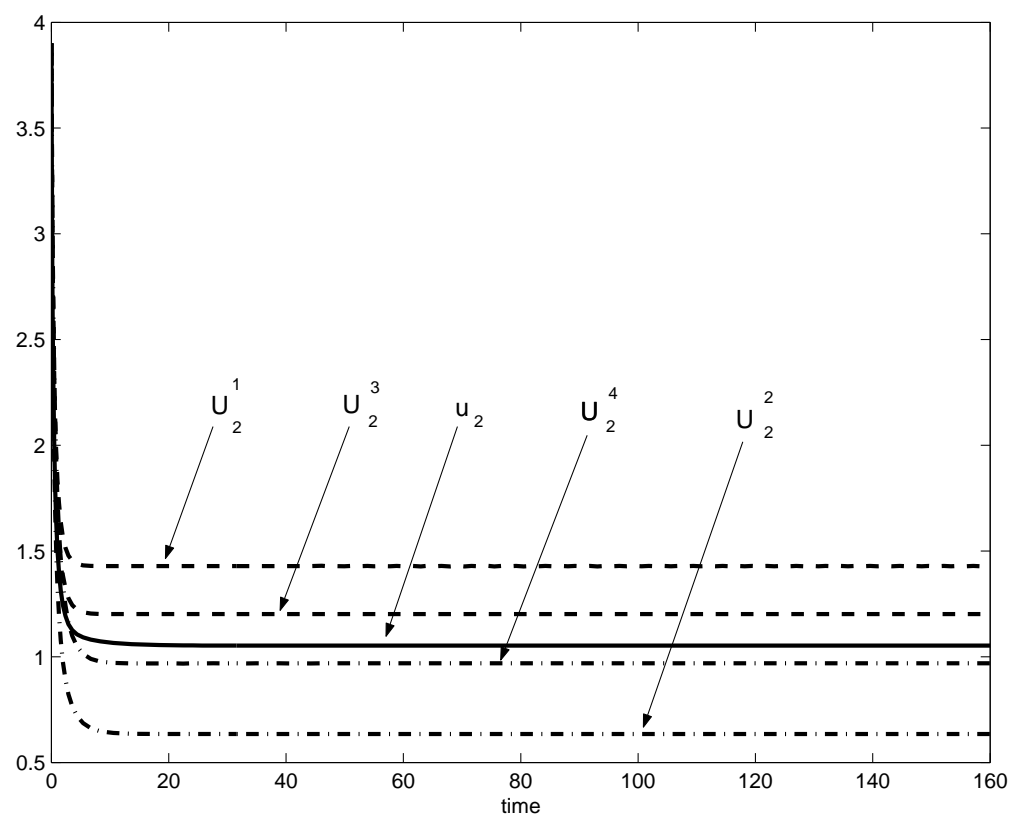

Fig. 2. Iterative scheme for $z^{0}=(1.4,3.9)$

\section{Discussion}

The sufficient conditions we proposed in the paper make it possible to develop an iterative scheme for a competitive Lotka-Volterra system, providing a sequence of observer systems for the localization of the solution of the original system. Although theoretically only a convergence on compact intervals can be guaranteed, the numerical simulations confirm convergence on infinite time intervals, as well. Hence, in practice, by this method the long-term behaviour of the population system can also be analyzed.

Since the condition of partially monotony of the observation function is also fulfilled for phenotypic observation of genetic processes, calculated in terms of the Hardy-Weinberg proportions (see López et al. 2007a), an extension of the above methodology to frequency-dependent models of population genetics may be straightforward.

\section{Acknowledgment}

This publication has been realized in the framework of the Hungarian-Spanish intergovernmental scientific and technological collaboration, with the support of the Scientific and Technological Innovation Fund (of Hungary) and the Ministry of Education and Sciences (of Spain) HH2004-0013, and also supported by the Hungarian National Scientific Research Fund (OTKA K 62000, K 72339). 


\section{References}

[1] Carreño, R.; Gámez, M.; Granados, B. and Tineo, A., Continuos dependence of the global attractors of a family of periodic kolmogorov systems. Nonlinear Analysis: Real World Applications 3 (2002) 25-36.

[2] Chen, B.M.; Lin Z. and Shamash Y., Linear Systems Theory. A Structural Decomposition Approach, Birkhauser, Boston (2004).

[3] Freedman, H. Deterministic Mathematical models in population ecology. Marcel Dekker, New York, (1980).

[4] Gámez, M., Modelo depredador-presa. Aplicaciones al control biológico. University of Almería. Doctoral Thesis. (1999), 134 pp.

[5] López, I.; Gámez, M.; Garay, J. and Varga Z., Monitoring in a Lotka-Volterra model. Biosystems 87 (2007), 68-74.

[6] López, I.; Gámez, M. and Varga Z., Observer design for phenotypic observation of genetic processes. Nonlinear Anal.: Real World Appl. (2007), (in press).

[7] López, I.; Gámez, M. and Carreño, R., Observability in dynamic evolutionary models. Applied Mathematics Letter (2007), accepted.

[8] Lotka, A.J., Elements of physical biology. Williams and Wilkins, Baltimore, Maryland, USA, (1925).

[9] Scudo, F. and Y. R. Ziegler., The Golden Age of Theoretical Ecology. Springer Verlag, Berlin-Heidelberg-New York, (1978).

[10] Sundarapandian, V., Local observer design for nonlinear systems, Mathematical and computer modelling, 35 (2002), 25-36.

[11] Tineo, A., Iterative schemes for some population models, Nonlin. World, 3 (1996), 695-708.

[12] Tineo, A., Existence of Coexistence States: A Generic Property for Cyclic 3Dimensional Competitive Systems. J. Math. Anal. Appl. 258 (2001), 13-21.

[13] Volterra, V., em Leçons sur la théorie mathématique de la lutte pour la vie. Gauthier-Villars, Paris, (1931).

[14] Yodzis, P., Introduction to Theoretical Ecology. Harper and Row. New York, (1989). 